\title{
Actin Reorganization in Hippocampal Neurons May Play Roles in Early Impairment of Learning and Memory in Rats After Propofol Anesthesia
}

\section{Xuena Zhang}

Beijing Chaoyang Hospital

Jie Li

Beijing Chaoyang Hospital

ANSHI WU ( $\sim$ sn-xn@163.com )

Beijing Chao-yang Hospital, Capital medical university https://orcid.org/0000-0002-3288-9217

Research article

Keywords: Lerning and memory, Anesthesia, Actin, Reorganization

Posted Date: June 3rd, 2020

DOI: https://doi.org/10.21203/rs.3.rs-30634/v1

License: (c) (1) This work is licensed under a Creative Commons Attribution 4.0 International License.

Read Full License 


\section{Abstract}

Background Perioperative neurocognitive disorder (PND) is a kind of neuronal complication especially observed in elderly patients. The present study was conducted to observe the changes of actin in hippocampus after propofol anesthesia, and to evaluate their roles in consequent learning impairment in both young (3-month-old) and aged (20-month-old) male rats.

Methods Double-shuttle box was used to learning evaluation since1, 3, 7 or $14 \mathrm{~d}$ after anesthesia. Hippocampi were removed after evaluations. F-actin content and the spines were observed through immunofluorescence and laser scanning confocal microscope (LSCM).

Results In young rats, latency of escape response (LER) was prolonged within 3 days after anesthesia. However, LER was significantly prolonged until 7days after anesthesia in elderly rats. Moreover, the learning curves were also shift in old rats. Dendritic spines became smaller in anesthesia groups of aged rats within 3 days, where the F-actin contents were significantly increased until 14d post anesthesia.

Conclusion Our results indicate that learning ability could be inhibited until after propofol anesthesia especially in old rats. The over-polymerization of actin and the consequent reorganization of dendrite spines in hippocampus may be responsible, which provides fresh evidence in aspect of synaptic plasticity for PND mechanism.

\section{Background}

Perioperative neurocognitive disorders (PND) have been discussed for over one hundred years. However, the mechanisms underlying are still unclear. There are many causes that may contribute to the occurrence of PND, which would finally induce the changes in synaptic plasticity and the consequent observable dysfunction in learning and memory [1].

For synaptic plasticity, two things are usually considered carefully,the structure of synapse and its function. It is known that the morphology of dendrite spines in the postsynaptic membrane reflects as well as take part in synaptic plasticity, which is mainly regulated by the dynamic transformation between G-actin and F-actin [2]. The long-term potentiation (LTP) and long-term depression (LTD) make the functional plasticity, both are fine tuned by multiple molecules. There are interplays between the structural and functional aspects. For example, more actins are polymerized during LTP [3], whereas Gactin and smaller spines are related to LTD [4].

Anesthetics have been found to have effects on synaptic plasticity. For example, propofol has been reported to inhibit LTP [5]. It was also found to be able to modulate actin dynamics [6]. How about the status of actin dynamic after anesthesia? No evidence has been provided although it may help to understand PND better. 
During our early exploration for the mechanism of PND, some actin binding proteins such as cofilin1 (CFL1) and coronin1a (CORO1A) were found differentially expressed in rat hippocampus after propofol anesthesia, implying the changes in actin dynamics [7]. Our further studies confirmed the above speculation through electrophysiologic and morphologic observations, where F-actin level was found increased before and during LTP induction in hippocampus of old rats 14 days after propofol anesthesia. At the same time, the amplitude of LTP was found decreased [8].

We postulated that the over-polymerized actin might contribute to occurrence of PND through disturbing the synaptic plasticity. In the present study, enhance actin polymerization and reorganized spines were observed. Both are related to PND in old rats after propofol anesthesia.

\section{Methods}

Animals

Male Sprague-Dawley rats of 3 months (young, $n=120$ ) and 20 months (aged, $n=120$ ) were purchased from Chengdu da shuo biotechnology co., LTD. All animal procedures were performed under the standard conditions in Beijing Chao-yang Hospital of Capital Medical University, and all experiments conformed animal care protocols of the institution. Young or aged rats were divided into five groups respectively, according to table of random numbers. Decapitation was used for rat euthanasia.

Anesthesia

All rats were housed individually at a constant room temperature $\left(20-22^{\circ} \mathrm{C}\right)$ and humidity $(50-60 \%)$ with a 12-h light/dark cycle. They were freely fed with a standard diet and tap water. To induce anesthesia, propofol of $100 \mathrm{mg} / \mathrm{kg}$ were injected intraperitoneally. Anesthesia status was kept with propofol at a rate of $50 \mathrm{mg} / \mathrm{kg} / \mathrm{h}$ through caudal vein for 3 hours. In control group, rats underwent the same procedure, but propofol was replaced by an equivalent volume of saline.

Rats undergoing propofol anesthesia with no respond to the tail pinch test were chosen for the following study. They were placed in a temperature-controlled incubator $\left(28^{\circ} \mathrm{C}\right)$ until the end of anesthesia. Arterial blood pressure and blood gases of propofol-treated rats were continuously measured and were kept in the normal range during anesthesia. All rats were then housed before further experiments.

\section{Behavioral experiment}

Shuttle box was used for learning and memory measurement. Training for rats started since 1, 3, 7 or $14 \mathrm{~d}$ after the end of anesthesia between 8AM and 12AM and continued for 7 days. Protocols were controlled through computer. The latency of escaping response (LER), active avoidance reaction (AAR), and passive avoidance reaction (PAR) were examined and compared on the 7th day after training.

F-actin content and spines 
Rats were sacrificed and brains were removed after training and testing. Brains were fixed by $4 \%$ formalin for 12 hours, and were then put into $20-30 \%$ sucrose for gradient dehydration. The dehydrated brains were kept in $30 \%$ sucrose with sodium azide or were sectioned into slices of $10-1 \mathrm{~m}$ thickness for further experiments through a microtome (CM1860; Leica, Nussloch, Germany).

Slices were incubated with a rabbit antibody against postsynaptic density protein 95 (PSD-95, ab18258, Abcam, USA) or a rabbit anti rat antibody against microtubule associated protein-3 (MAP-2, ab32454 abcam, USA ) at $4^{\circ} \mathrm{C}$ for 40 hours. After that, slices were washed with $1 \%$ phosphate buffered saline (PBS; HyClone) for three times, and were incubated with Alexa Fluor 488-labeled goat anti-rabbit $\operatorname{lgG}(1: 500)$ for $15 \mathrm{~min}$ at room temperature. For F-actin observation, a mouse anti rat F-actin-NH3 (ab205,

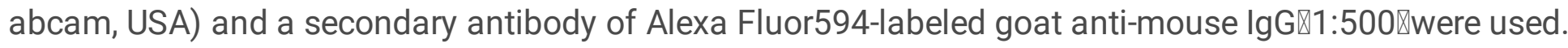
All slices were washed by PBS for 3 times and were viewed under a laser confocal microscopy (BX53; Olympus, Tokyo, Japan).

Statistic Analysis

Protein fluorescent densities were evaluated by one-way analysis of variance (ANOVA) followed by the Bonferroni post hoc test using SPSS version 17.0 (SPSS, Chicago, IL, USA). A P value of less than 0.05 was considered statistically significant.

\section{Results}

There were no significant differences in rat weights and amounts among groups (12/12 in all young rat groups, $11 / 12$ in D1 and D 14, 10/12 in D3, and 12/12 in D7 groups of aged rats.)

Behavioral experiment

Our data showed that the learning abilities in old rats were inhibited. Also the LER of old rats were prolonged until 7days after anesthesia and were back to normal level within 14 days $(P<0.05)$ (Fig. 1). For young rats, similar changes were only detected within 3days after anesthesia. Moreover, LER of old rats was significantly longer than that of young rats at every time point (Fig. 2).

Dendrite spine morphology

More spines in hippocampal neurons were observed after shuttle box training, with longer and slimmer terminals. After anesthesia however, the amount of spines were found decreased, with obvious contractions in length, especially within 3 days in old rats. Dendrite spines of young rats were also shortened within 3 days after anesthesia, without significant changes in morphology (Fig. 3).

F-actin content in hippocampal neurons

More F-actins were observed in young rats than in old rats before anesthesia $(P<0.05)$. F-actins contents were increased after shuttle box training in all rats. They were also up-regulated after anesthesia, which 
continued until the 7th day in young rats and until the 14th day in old rats (Table 1, Fig. 4).

Table 1

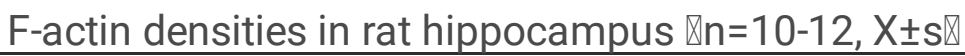

\begin{tabular}{|c|c|c|}
\hline Groups & $Y$ & 0 \\
\hline C & $102.06 \pm 10.18$ & $70.14 \pm 7.72^{\mathrm{ab}}$ \\
\hline D & $156.15 \pm 12.97^{b}$ & $91.13 \pm 8.62^{\mathrm{ab}}$ \\
\hline$A_{1}$ & $488.01 \pm 29.15^{b}$ & $569.81 \pm 33.63^{a b}$ \\
\hline $\mathrm{A}_{3}$ & $294.88 \pm 20.63^{b}$ & $379.23 \pm 22.57^{\mathrm{ab}}$ \\
\hline$A_{7}$ & $212.83 \pm 18.81^{b}$ & $350.63 \pm 23.76^{a b}$ \\
\hline $\mathrm{A}_{14}$ & $114.63 \pm 9.65$ & $239.77 \pm 25.81^{\mathrm{ab}}$ \\
\hline $\mathrm{AD}_{1}$ & $416.03 \pm 24.33^{b}$ & $454.54 \pm 24.86^{\mathrm{ab}}$ \\
\hline $\mathrm{AD}_{3}$ & $389.43 \pm 27.30^{b}$ & $426.22 \pm 20.55^{\mathrm{ab}}$ \\
\hline$A D_{7}$ & $581.04 \pm 32.25^{b}$ & $579.61 \pm 30.28^{a b}$ \\
\hline $\mathrm{AD}_{14}$ & $152.77 \pm 11.64^{b}$ & $82.37 \pm 8.03^{\mathrm{ab}}$ \\
\hline \multicolumn{3}{|c|}{$\begin{array}{l}\text { C: control. D: shuttle box group. } A_{1,3,7,14} \text { : groups of } 1,3,7 \text {, or } 14 \text { days after anesthesia. } A D_{1,3,7,14} \text { : rats } \\
\text { start training since } 1,3,7 \text {, or } 14 \text { days after anesthesia. } Y \text { : young rat groups. } 0 \text { : old rat groups. }{ }^{a}: P<0.05 \\
\text { comparing with those of group } Y \text {. }{ }^{b}: P<0.05 \text { comparing with those of group } C \text {. ANOVA,F }=9.84, n=10- \\
12, S-N-K \text { पtest }\end{array}$} \\
\hline
\end{tabular}

\section{Discussion}

Advanced age has been accepted as one of the causes for PND [9], which is again confirmed by the present study. All the rats got AAR after training. However, the learning abilities of old rats were decreased, presented as the right-shifted learning curves. Moreover, the reactions of old rats are believed to decline because their LERs were longer than those of the young rats at every time point. The delayed recovery of reaction in old rats until 14 days after anesthesia were also observed comparing with those of the young rats returning to normal within 7days. All are the evidence for the susceptibility of senility to PND.

One of the differences between the young and old synapses is the morphology of dendrite spines. It is known that spines in old brain are usually short in length and are decreased in density, which is believed to contribute to the cognitive impairment [10]. In the present study, we found the difference of spines between young and old rats before and after anesthesia, which could explain the inhibited learning and memory capability of old rats to some degree, as well as suggest the important role of the changes in spine morphology in PND. 
The decreased, shortened, and contracted spines in hippocampus after anesthesia would indicate the inhibited synaptic plasticity. It is generally accepted that LTP and LTD are associated with long-term enlargement and shrinkage of dendritic spines respectively [11]. Moreover, the biochemical states inside spines could be dynamically modulated by synaptic activity, leading to the reorganization of cytoskeletal architecture, and then the plasticity of structure and function of the spine [12]. Propofol has been found to be able to decrease dendritic spine density and restrict the spine development in some studies, which were thought to be involved in the cognitive dysfunction during and after anesthesia [13]. Changes in dendrite spines observed in the present study should also be responsible for the inhibited learning and memory, which has been proven by the behavioral experiment. Therefore, we postulate that the modified spine shapes should be one of the morphological features in hippocampus of PND.

The actin cytoskeleton is the principal architectural component of the dendrite spine, whose dynamic remodeling, or the balance between F-actin and G-actin, plays a major role in structural plasticity of dendritic spines [14]. Proper polymerization of actin is the base of LTP [15]. Over polymerized actin are found to be associated with neural degeneration and cognitive dysfunction [16]. Previous studies have reported the reorganization of actin induced by propofol [6]. In one of our early research, we found increased F-actin level in old rat hippocampus after propofol anesthesia, which was closely related to the depression of LTP, indicating the important roles of overpolymerization of actin in synaptic dysfunction after propofol anesthesia[8].

There are many molecules that regulate the actin dynamics, some of which could be affected by propofol. We have reported that phosphorylated Cofilin (p-cofilin1) 1 was up-regulated during LTP induction in elderly rats after propofol anesthesia, which may be associated with the increased F-actin and depressed LTP8. It is known that Cofilin1 is the key regulator that usually conducts the depolymerization of actin and is inactive when it is phosphorylated [17]. Therefore, we postulate that the inactivation of Cofilin1 may play roles in the impairment of learning and memory in old rats after propofol anesthesia, but further study is still required.

\section{Conclusion}

In summary, our study revealed that the aged rats exerted a weaker resistance against propofol-induced cognitive dysfunction than young rats. The present differential results obtained from rats of different ages were at least partially associated with the reconstruction of dendrite spines and aberrant F-actin dynamic equilibrium in hippocampus, which may also be on of the possible explanations for the mechanism of PND.

\section{Abbreviations}

PND

Perioperative neurocognitive disorder

LSCM 
laser scanning confocal microscope

LER

latency of escape response

LTP

long-term potentiation

LTD

long-term depression

CFL1

cofilin1

COR01A

coronin1a

\section{Declarations}

\section{Ethics approval and consent to participate}

For the data used in this article, we have got the approval form the Ethics Committee of Beijing Chaoyang Hospital.

\section{Consent for publication}

Not applicable

\section{Availability of data and material}

The datasets used and/or analysed during the current study are available from the corresponding author on reasonable request.

\section{Competing interests}

Not applicable

\section{Funding}

This study was supported by National Natural Science Foundation of China, code: 81000473. Xuena Zhang was the recipient of this funding. The protocol, performance, and quality control were supervised by pro. Anshi Wu. He was also responsible for the data analysis. Jie Li was the major researcher of this funding.

\section{Author's contributions}


XZ designed this project, and was responsible for the LSCM experiment and data analysis. JL was responsible for the behavioral test and related data analysis.

AW is the corresponding author. He is responsible for the interpretation and statistical analysis of data.

All authors have read and approved the manuscript. Acknowledgements

Not applicable

\section{References}

1. Qiu LL, Pan W, Luo D, Zhang GF, Zhou ZQ, Sun XY, Yang JJ, Ji MH. Dysregulation of BDNF/TrkB signaling mediated by NMDAR/ $\mathrm{Ca}(2+) /$ calpain might contribute to postoperative cognitive dysfunction in aging mice. J Neuroinflammation. 2020;17(1):23.

2. Borovac J, Bosch M, Okamoto K. Regulation of actin dynamics during structural plasticity of dendritic spines: Signaling messengers and actin-binding proteins. Mol Cell Neurosci. 2018;91:12230.

3. Borovac J, Bosch M, Okamoto K. Regulation of actin dynamics during structural plasticity of dendritic spines: Signaling messengers and actin-binding proteins. Mol Cell Neurosci. 2018;91:12230.

4. Szabó EC, Manguinhas R, Fonseca R. The interplay between neuronal activity and actin dynamics mimic the setting of an LTD synaptic tag. Sci Rep. 2016;6:33685.

5. Sun M, Yuan R, Liu H, Zhang J, Tu S. The effects of repeated propofol anesthesia on spatial memory and long-term potentiation in infant rats under hypoxic conditions. Genes Dis. 2019;7(2):245-52.

6. Pearn ML, Schilling JM, Jian M, Egawa J, Wu C, Mandyam CD, Fannon-Pavlich MJ, Nguyen U, Bertoglio J, Kodama M, Mahata SK, DerMardirossian C, Lemkuil BP, Han R, Mobley WC, Patel HH, Patel PM, Head BP. Inhibition of RhoA reduces propofol-mediated growth cone collapse, axonal transport impairment, loss of synaptic connectivity, and behavioural deficits. Br J Anaesth. 2018;120(4):745-60.

7. Zhang $X$, Yang $S$, Yue Y, Wu A. Profiling of the soluble proteome in rat hippocampus post propofol anesthesia. Neurochem Res. 2013;38(12):2661-7.

8. Li M, Zhang X, Wu A, Wang Z, Li J, Yue Y. Propofol-induced age-different hypocampal long-term potentiation is associated with F-Actin polymerization in rats. Cell Biochem Biophys. 2015;71(2):1059-66.

9. Rengel KF, Pandharipande PP, Hughes CG. Special Considerations for the Aging Brain and Perioperative Neurocognitive Dysfunction. Anesthesiol Clin. 2019;37(3):521-36.

10. Xu B, Sun A, He Y, Qian F, Xi S, Long D, Chen Y. Loss of thin spines and small synapses contributes to defective hippocampal function in aged mice. Neurobiol Aging. 2018;71:91-104. 
11. Chidambaram SB, Rathipriya AG, Bolla SR, Bhat A, Ray B, Mahalakshmi AM, Manivasagam T, Thenmozhi AJ, Essa MM, Guillemin GJ, Chandra R, Sakharkar MK. Dendritic spines: Revisiting the physiological role. Prog Neuropsychopharmacol Biol Psychiatry. 2019;92:161-93.

12. Nakahata Y, Yasuda R. Plasticity of Spine Structure: Local Signaling, Translation and Cytoskeletal Reorganization. Front Synaptic Neurosci. 2018;10:29.

13. Zhang S, Liang Z, Sun W, Pei L. Repeated propofol anesthesia induced downregulation of hippocampal miR-132 and learning and memory impairment of rats. Brain Res. 2017;1670:156-64.

14. Borovac J, Bosch M, Okamoto K. Regulation of actin dynamics during structural plasticity of dendritic spines: Signaling messengers and actin-binding proteins. Mol Cell Neurosci. 2018;91:12230.

15. Borovac J, Bosch M, Okamoto K. Regulation of actin dynamics during structural plasticity of dendritic spines: Signaling messengers and actin-binding proteins. Mol Cell Neurosci. 2018;91:12230 .

16. Woolfrey KM, Srivastava DP. Control of Dendritic Spine Morphological and Functional Plasticity by Small GTPases. Neural Plast. 2016; 2016:3025948.

17. Chen B, Wang Y. Cofilin rod formation in neurons impairs neuronal structure and function. CNS Neurol Disord Drug Targets. 2015;14(4):554-60.

\section{Figures}

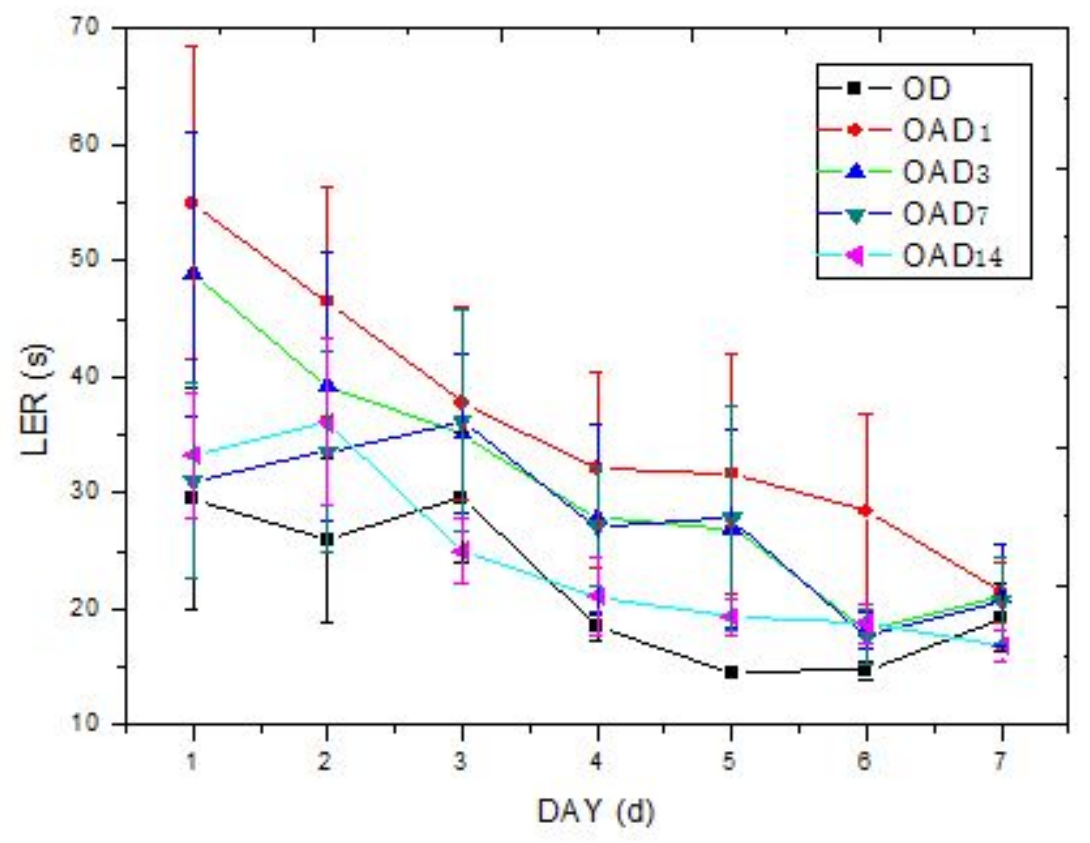

Figure 1 
Learning curves of old rats. OD: old rats only for shuttle box training. OAD: old rats starting shuttle box training since 1 day(OAD1), 3days(OAD3), 7days(OAD7), or 14days(OAD14) after propofol anesthesia. Comparing with that of OD, curves in OAD1区OAD3 and OAD7 were up shifted and LERs were prolonged $(P<0.05)$.

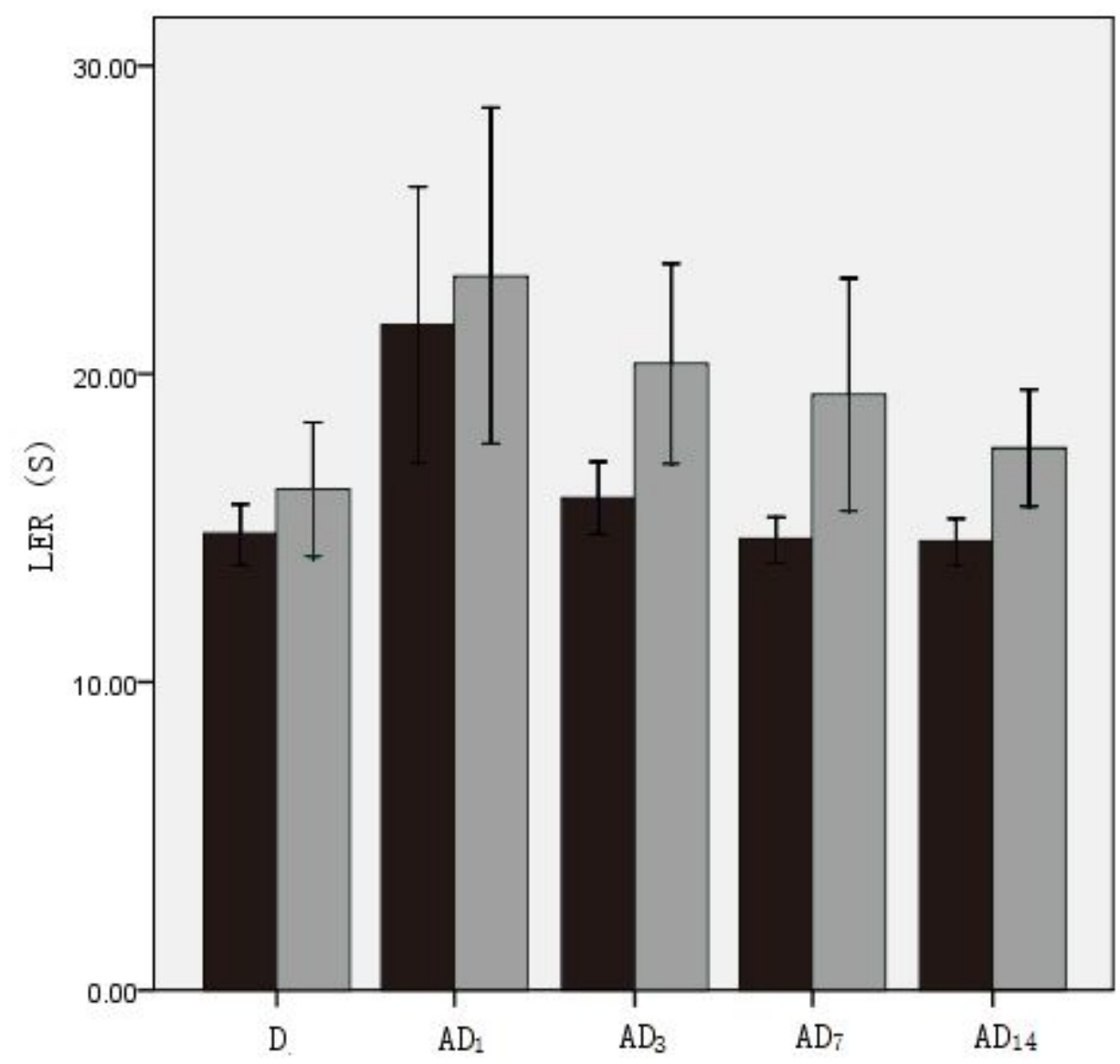

$\mathrm{A}$
$\square \mathrm{B}$

\section{Figure 2}

LER comparation between the young and old rats. A: young rat. B: old rats. D: rats for shuttle boxing training only. $A D$ : rats starting shuttle box training since 1day(AD1), 3days(AD3), days (OAD7), or 14days (AD14) after propofol anesthesia. LERs of old rats were longer than those of the young rats at every time point. $(\mathrm{P}<0.05)$. 

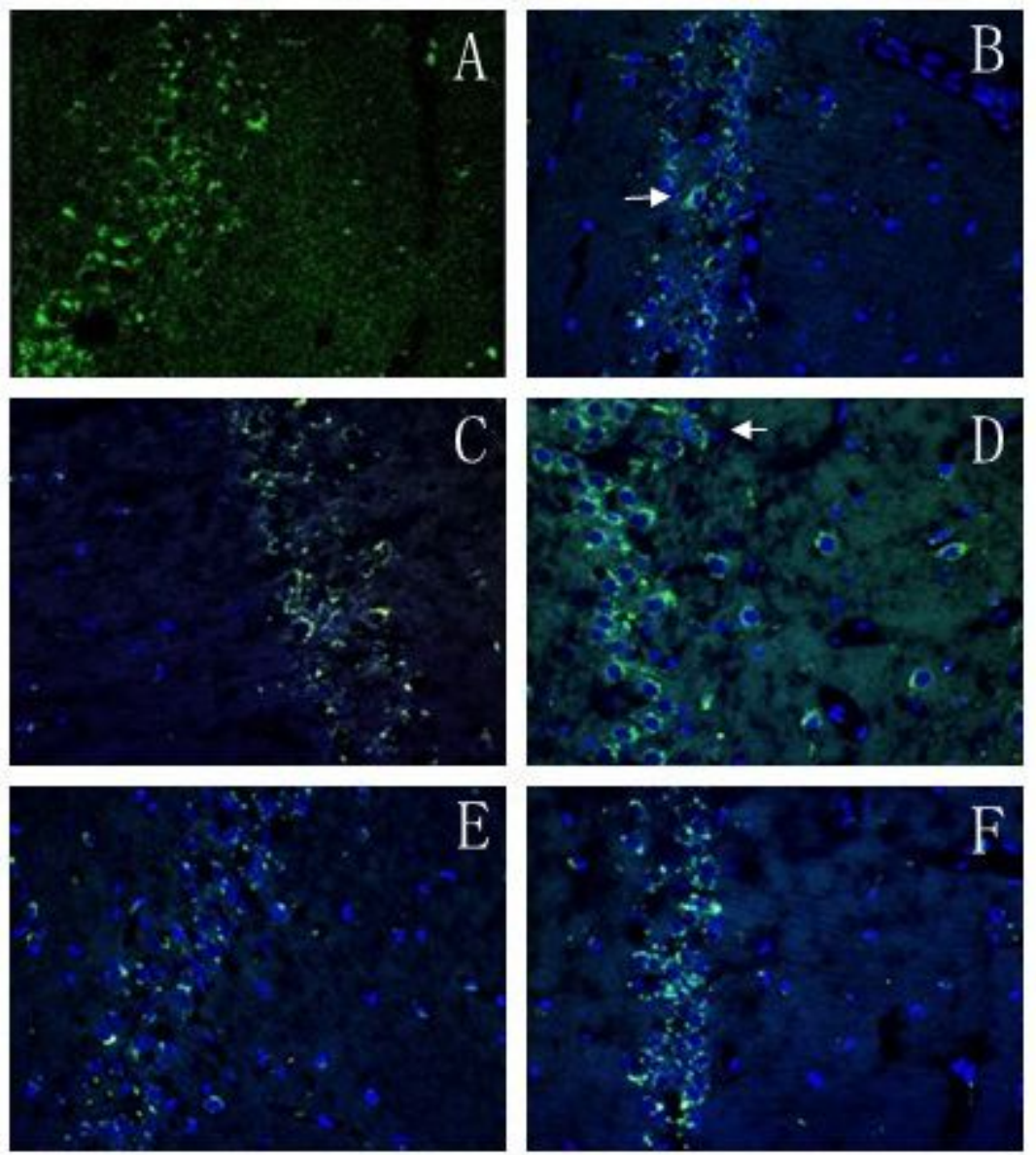

\section{Figure 3}

Dendrite spines labeled with AlexaFluor596 in hippocampus of old rats through LSCM. A control. B shuttle box group. C 1d after anesthesia. D 14d after anesthesia. E starting shuttle box training $1 \mathrm{~d}$ after anesthesia. F starting shuttle box training $7 \mathrm{~d}$ after anesthesia. 

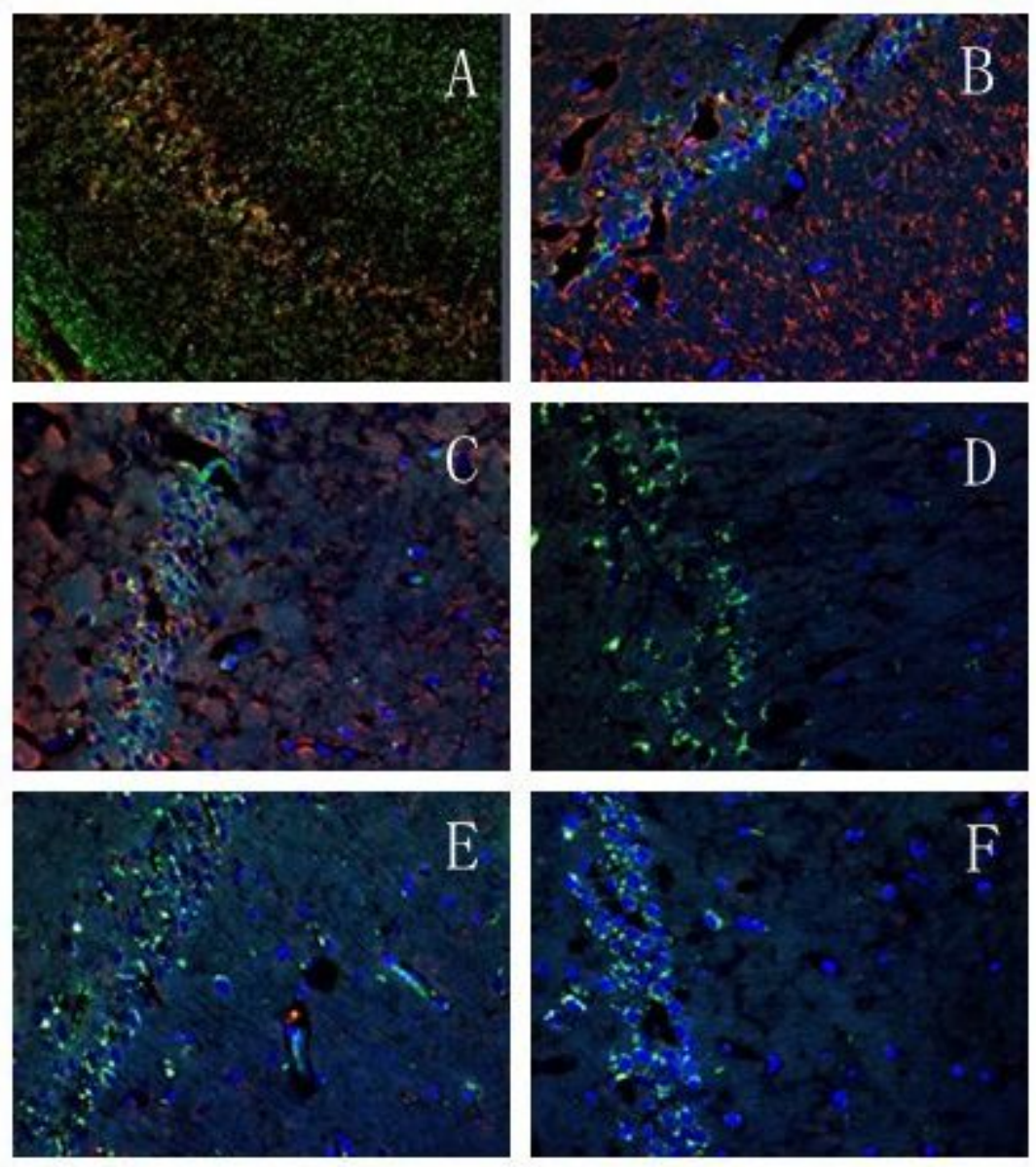

Figure 4

F-actin labeled with AlexaFluor488b (red) and MAP2 labeled with AlexaFluor596 (green) in hippocampus of old rats. A control. B shuttle box group. C $1 \mathrm{~d}$ after anesthesia. D 14d after anesthesia. E starting shuttle box training $1 \mathrm{~d}$ after anesthesia. $\mathrm{F}$ starting shuttle box training $7 \mathrm{~d}$ after anesthesia.

\section{Supplementary Files}

This is a list of supplementary files associated with this preprint. Click to download.

- NC3RsARRIVEGuidelinesChecklistfillable.pdf 